\title{
Edgewise Subdivision of a Simplex *
}

\author{
Herbert Edelsbrunner $^{\dagger}$ and Daniel R. Grayson ${ }^{\ddagger}$
}

\begin{abstract}
In this paper we introduce the abacus model of a simplex and use it to subdivide a $d$-simplex into $k^{d} d$-simplices all of the same volume and shape characteristics. The construction is an extension of the subdivision method of Freudenthal [4].
\end{abstract}

Keywords. Mesh generation, subdivision, tiling, simplex shape, symmetry.

\section{Introduction}

It is easy to subdivide a triangle into four similar triangles all of the same area: cut each edge into equal halves and connect the three dividing points. Since the triangles are similar we can repeat the operation and refine while preserving the triangle shape. Such a construction does not exist in general for tetrahedra and higher-dimensional simplices.

Result. A subdivision of a $d$-simplex is a decomposition into $d$-simplices such that each pair is either disjoint or meets along a common face. In other words, the $d$-simplices and their faces form a simplicial complex. Such complexes are used in engineering and science to represent geometric shapes and domains for the purpose of design, analysis, simulation and visualization.

\footnotetext{
${ }^{*}$ Research by both authors is partially supported by NSF under grant DMS 98-73945. Research of the first author is also supported by NSF under grant CCR-96-19542, and by ARO under grant DAAG55-98-1-0177.

tDepartment of Computer Science, University of Illinois at Urbana-Champaign, Urbana, Illinois 61801, and Raindrop Geomagic, Champaign, Illinois 61820.

$\ddagger$ Department of Mathematics, University of Illinois at UrbanaChampaign, Urbana, Illinois 61801.
}

Permission to make digital or hard copies of all or part of this work for personal or classroom use is granted without fee provided that copies are not made or distributed for profit or commercial advantage and that copies bear this notice and the full citation on the first page. To copy otherwise, to republish, to post on servers or to redistribute to lists. requires prior specific permission and/or a fee

SCG'99 Miami Beach Florida

Copyright ACM 1999 1-58113-068-6/99/06 ..\$5.00
The main result of this paper is a generalization of the triangle 4-division mentioned above that preserves most of the symmetry found in the two-dimensional case.

Main Theorem. For every integer $k \geq 1$ and every $d$ simplex $\sigma$ there is a subdivision into $k^{d} d$-simplices $\sigma_{i}$ with the following properties:

(1) all $\sigma_{i}$ have the same $d$-dimensional volume,

(2) the $\sigma_{i}$ fall into at most $\frac{d !}{2}$ congruence classes,

(3) the faces of $\sigma$ are subdivided the same way,

(4) repeated subdivision has the same effect as increasing $k$,

(5) except for boundary effects the neighborhoods of vertices are translates of each other.

Figures 4 through 7 show subdivisions satisfying the Main Theorem. Properties (1) through (5) are statements of symmetry. Observe that the number of congruence classes in Property (2) is independent of $k$, where two simplices belong to the same class iff one is obtained from the other by a combination of translations, rotations, and reflections. Property (3) means that the effect of the subdivision on a face of $\sigma$ is exactly the subdivision of that face with the same $k$ and the same method. Property (4) says that instead of subdividing each $\sigma_{i}$ into $\ell^{d} d$-simplices we can subdivide $\sigma$ into $(k \ell)^{d} d$-simplices and reach the same result. Property (5) implies that we can think of the subdivision as the intersection of $\sigma$ with a periodic tiling of $\mathbb{R}^{d}$ that looks the same from every vertex.

Previous and related work. For $k=2$, the subdivisions of the Main Theorem have been described by Freudenthal in 1942 [4]. In contrast to our algebraic approach, his construction is geometric, as are the related triangulations of the $d$-dimensional cube by Coxeter [2] and Kuhn [6]. The 2-dimensional case of Freudenthal's subdivision is the 4-division of the triangle illustrated in Figure 4. It is frequently used in the generation of 2-dimensional grids as well as the modeling of surfaces 
in space. The 3-dimensional case is the 8-division of the tetrahedron; see Figure 5. It was studied by Bey [1] and by Liu and Joe [7] in the context of adaptive mesh refinement for finite element analysis. Freudenthal's subdivision for general dimensions was also considered by Moore [8] who studied hierarchies for adaptive meshing. The work in this paper takes its inspiration from the algebraic constructions of Grayson [5]. He subdivides simplices into Cartesian products of simplices in the context of K-theory. This approach leads to a simple and purely algebraic description of simplices and subdivisions that is amenable to computer implementations.

Apart from mesh refinement for finite element analysis we see applications of our results in computer graphics. The combination of the 4-division of a triangle with a weighted averaging of vertex positions has emerged as a popular tool for surface modeling, see for example DeRose et al. [3]. The generalization of this method to the manipulation and display of 3and higher-dimensional density distributions requires an easy to compute periodic subdivision that does not suffer from shape deterioration as the simplices get smaller. The Main Theorem offers a solution to this problem.

Outline. Section 2 describes an algorithm that maps a point to a simplex containing the point. Section 3 proves the Main Theorem by collecting the simplices generated by the algorithm. Scction 4 illustrates and discusses sample subdivisions in dimensions $2,3,4$. Section 5 concludes the paper.

\section{Abacus Model of a Simplex}

We think of a $d$-simplex primarily as a sequence of $d+1$ numbers in the unit interval. With the introduction of this idea we prepare an algebraic interpretation of a simplex that frees us from depending on our geometric intuition.

Colored rectangle. Let $P_{0}, P_{1}, \ldots, P_{d}$ be affinely independent points that span a simplex $\sigma$ in $\mathbb{R}^{d}$. The ordering of the points is important, as it affects the following construction. If $b_{0}, b_{1}, \ldots, b_{d}$ are non-negative real numbers that sum to 1 then

$$
X=\sum_{i=0}^{d} b_{i} \cdot P_{i}
$$

is a point in $\sigma$. The $b_{i}$ are the barycentric coordinates of $X$. We may portray them graphically by drawing the unit interval as a rectangle with regions colored from 0 .through $d$, making sure to arrange the colors from left to right, so that $b_{i}$ is the fraction of points with color $i$. Figure 1 illustrates this for $d=7$ and the point with barycentric coordinates

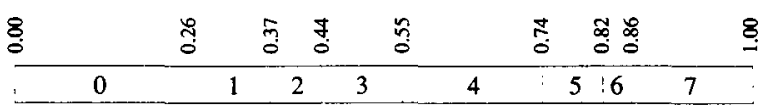

Figure 1: The rectangle represents the unit interval with points colored from 0 through 7 .

$(0.26,0.11,0.07,0.11,0.19,0.08,0.04,0.14)$. The coordinates of the dividing lines are displayed above the rectangle. They are provided by the partial sums $0=$ $B_{0}, B_{1}, \ldots, B_{d}, B_{d+1}=1$ with $B_{i}=b_{0}+b_{1}+\ldots+b_{i-1}$. $B_{1}$ through $B_{d}$ can be any non-decreasing sequence in the unit interval.

Stack of rectangles. Suppose we chop the rectangle in Figure 1 into $k$ pieces of equal width, stack them on top of cach other, and expand the scalc by a factor of $k$ in the horizontal direction for clarity; see Figure 2 . The coordinates of the dividing lines are obtained by

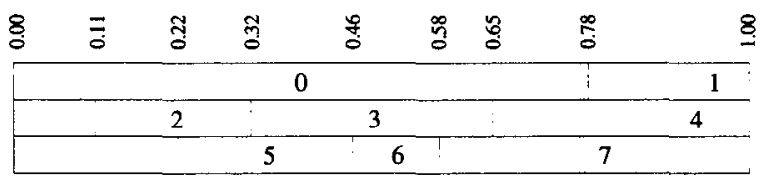

Figure 2: The rectangle in Figure 1 is chopped into three pieces. which are stacked and expanded.

multiplying the coordinates of the earlier dividing lines by $k$ and discarding the integer part, keeping only the fractional part. We discard any duplicates, letting $j+2$ be the number of distinct values remaining. Call these numbers $0=C_{0}, C_{1}, \ldots, C_{j}, C_{j+1}=1$, making sure to sort them in increasing order. We extend the dividing lines vertically until they span the entire stack and label each region by its color; see Figure 3 . The widths of the regions in the stack are $c_{i}=C_{i+1}-C_{i}$, for $0 \leq i \leq j$.

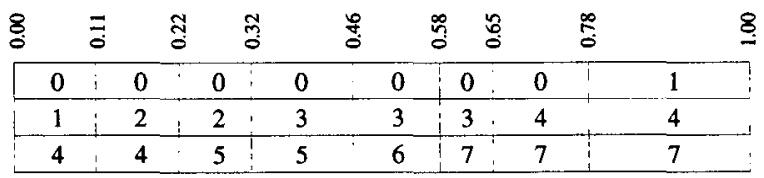

Figure 3: Each rectangle is cut into equally many regions, and each region keeps the original color of its points.

Color scheme. The number of regions in each row of the stack is $j+1$. Forgetting the positions of the vertical dividing lines we get a matrix

$$
\chi=\left(\begin{array}{cccc}
\chi_{1,0} & \chi_{1,1} & \ldots & \chi_{1, j} \\
\chi_{2,0} & \chi_{2,1} & \ldots & \chi_{2, j} \\
\vdots & \vdots & \ddots & \vdots \\
\chi_{k, 0} & \chi_{k, 1} & \ldots & \chi_{k, j}
\end{array}\right)
$$


of $k(j+1)$ colors. We call $\chi$ a color scheme because it determines the combinatorics but not the geometry of the coloring. The matrices that may occur are those whose entries are drawn from the set $\{0,1, \ldots, d\}$, whose entries are in non-decreasing order when read like text:

$$
\chi_{1,0} \leq \chi_{1,1} \leq \ldots \leq \chi_{1, j} \leq \chi_{2,0} \leq \ldots \leq \chi_{k, j},
$$

and whose columns are pairwise different.

Combination of points. The numbers $c_{i}$ sum to 1 and can therefore be used as barycentric coordinates to express our original point $X$ in terms of other points. For $k$ not necessarily distinct colors $\chi_{1}$ through $\chi_{k}$ we introduce the notation

$$
P_{\chi_{1} \chi_{2} \ldots \chi_{k}}=\left(P_{\chi_{1}}+P_{\chi_{2}}+\ldots+P_{\chi_{k}}\right) / k .
$$

This way we obtain exactly all points in our $d$-simplex $\sigma$ whose barycentric coordinates are integer multiples of $1 / k$. Each column in a color scheme corresponds to such a point. We now show that the $c_{i}$ are the barycentric coordinates of $X$ with respect to the points that correspond to the columns of the color scheme $\chi=\chi(X, k)$.

Combination Lemma. $X=\sum_{i=0}^{j} c_{i} \cdot P_{\chi_{1, i} \chi_{2, i} \ldots \chi_{k, i}}$.

Proof. The product $k \cdot b_{i}$ is the total width of regions with color $i$. It follows that the sum, over all entries of the color scheme, of $P_{i} / k$ times the width of the corresponding region is the sum of $b_{i} \cdot P_{i}$. By definition this is our original point $X$. Now consider

$$
\begin{aligned}
Y & =\sum_{i=0}^{j} c_{i} \cdot P_{\chi_{1, i} \chi_{2, i} \ldots \chi_{k, i}} \\
& =\frac{1}{k} \cdot \sum_{i=0}^{j} c_{i} \cdot\left(P_{\chi_{1, i}}+P_{\chi_{2, i}}+\ldots+P_{\chi_{k, i}}\right),
\end{aligned}
$$

which is a sum over all entries of the same color scheme. Each term corresponds to a column, and the contribution of an entry with color $i$ is again $P_{i} / k$ times the width of the corresponding region. Hence $X=Y$.

Independence of points. We prove that the points in the Combination Lemma are affinely independent. This implies that a color scheme with $j+1$ columns specifies a $j$-simplex. The color scheme depends on the sequence in which the vertices of $\sigma$ are presented. This sequence defines a directed path obtained by concatenating the shape vectors $V_{i}=P_{i}-P_{i-1}$ for $1 \leq i \leq d$. Since $\sigma$ is non-degenerate, the $d$ shape vectors are linearly independent.

Independence Lemma. The points $P_{\chi_{1, i} \chi_{2, i} \ldots \chi_{k, i}}$ defined by the columns of a color scheme $\chi$ are affinely independent.
Proof. Assume first that $\chi$ has $d+1$ columns and consider the vectors connecting points of adjacent columns:

$$
V_{i}^{\prime}=P_{\chi_{1, i} \chi_{2, i} \cdots \chi_{k, i}}-P_{\chi_{1, i-1} \chi_{2, i-1} \cdots \chi_{k, i-1}} .
$$

Because the color scheme is full, the transition from column $i-1$ to column $i$ changes only a single color, and that color increases by 1 . Suppose that the color increases in row $\ell$. We define a permutation $\pi$ of $\{1,2, \ldots, d\}$ by setting $\pi(i)=\chi_{\ell, i}=\chi_{\ell, i-1}+1 . \pi$ is indced a permutation because the transition to $\pi(i)$ occurs exactly once in the matrix $\chi$. We find that

$$
\begin{aligned}
V_{i}^{\prime} & =\frac{1}{k} \cdot\left(P_{\pi(i)}-P_{\pi(i)-1}\right) \\
& =\frac{1}{k} \cdot V_{\pi(i)} .
\end{aligned}
$$

The linear independence of the $V_{i}$ thus implies the linear independence of the $V_{i}{ }^{\prime}$, which implies the affine independence of the points $P_{\chi_{1, i} \chi_{2, i} \ldots \chi_{k, i}}$. A color scheme with $j+1$ columns is obtained by removing $d-j$ of the $d+1$ columns of a full color scheme. The corresponding operation for the vectors is addition. Specifically, the $d$ linearly independent vectors are grouped into $j$ sums, which are again linearly independent vectors and thus define a $j$-simplex.

\section{Subdivision of a Simplex}

Section 2 described an algorithm which takes a point of $\sigma$ and produces a smaller simplex within $\sigma$ that contains the point. We use that algorithm to construct a subdivision of $\sigma$.

Edgewise subdivision. Each point $X \in \sigma$ defines a unique color scheme $\chi=\chi(X, k)$ with $k$ rows. This color scheme defines a unique simplex $\sigma_{\chi}$, namely the one spanned by the points $P_{\chi_{1, i} \chi_{2, i} \ldots \chi_{k, i}}$ that correspond to the columns of $\chi$. The edgewise subdivision of $\sigma$ consists of all simplices defined this way by points of $\sigma$ :

$$
\operatorname{Esd}_{k} \sigma=\left\{\sigma_{\chi} \mid \chi=\chi(X, k), X \in \sigma\right\} .
$$

If we delete one or more columns from $\chi$ we get another color scheme $\chi(Y, k)$, where $Y$ is obtained from $X$ by setting one or more of the barycentric coordinates to zero and increasing the others accordingly. It follows that for each simplex $\sigma_{\chi}$ the edgewise subdivision contains all faces of that simplex. A set of simplices with that property is a subdivision of $\sigma$ iff the interiors of the simplices are pairwise disjoint and the union of interiors is $\sigma$. We establish the latter two properties for $\operatorname{Esd}_{k} \sigma$.

Subuivision Lemma. For each $k \geq 1, \operatorname{Esd}_{k} \sigma$ is a subdivision of $\sigma$. 
ProOF. The algorithm in Section 2 is deterministic and maps a point $X \in \sigma$ to a set of barycentric coordinates that express $X$ in terms of the vertices of a $j$-simplex $\sigma_{\chi} \subseteq \sigma$. These coordinates are positive, which implies that $X$ lies in the interior of $\sigma_{\chi}$. It follows that the union of interiors of simplices in $\operatorname{Esd}_{k} \sigma$ is $\sigma$.

What happens if we start at the other end? Suppose we take a $j$-simplex $\sigma_{\chi} \in \operatorname{Esd}_{k} \sigma$, choose non-zero barycentric coordinates $c_{0}+c_{1}+\ldots+c_{j}=1$ and define

$$
Y=\sum_{i=0}^{j} c_{i} \cdot P_{\chi_{1, i} \chi_{2, i} \cdots \chi_{k, i}} .
$$

Applying our algorithm to this point $Y$ will lead to the same $c_{i}$ and $\sigma_{\chi}$ because each step in the algorithm is uniquely reversible. It follows that the interiors of the simplices in $\operatorname{Esd}_{k} \sigma$ are pairwise disjoint.

Number of simplices. To count simplices we exploit the one-to-one correspondence between simplices and color schemes. For simplices of maximum dimension this is straightforward.

Counting Lemma. If the dimension of $\sigma$ is $d$ then the number of $d$-simplices in Esd $\mathrm{E}_{k} \sigma$ is $k^{d}$.

Proof. We may count the simplices in Esd ${ }_{k} \sigma$ by looking at the vertical dividing lines as illustrated in Figure 2. The color schemes are completely determined by working from left to right, and selecting for each dividing line the row in which it appears. For a $d$-simplex there are $d$ dividing lines, and each one may appear in one of $k$ rows, so there are $k^{d}$ possibilities.

To count simplices of dimension $j<d$ is similar but more complicated. Now we have $j$ dividing lines and each may be multiply defined. Furthermore, the right ending line of the stack may also be multiply defined. The number of $j$-simplices in $\operatorname{Esd}_{k} \sigma$ is therefore equal to the number of ways we can draw $d$ not necessarily distinct rows from $\{1,2, \ldots, k\}$ and distribute them over the $j$ dividing lines plus one right ending line in such a way that each dividing line receives at least one row.

Volume. For a simplex $\sigma$ with vertices $P_{0}, P_{1}, \ldots, P_{d}$ in $\mathbb{R}^{d}$ it is known that the $d$-dimensional volume is

$$
\operatorname{vol} \sigma=\frac{1}{d !} \cdot\left|\operatorname{det}\left(P_{1}-P_{0}, P_{2}-P_{0}, \ldots, P_{d}-P_{0}\right)\right| \text {. }
$$

We prove Property (1) of the Main Theorem, which claims that all $d$-simplices in the edgewise subdivision have the same volume.

MEASURING LEMma. The $d$-dimensional volume of every $d$-simplex $\sigma_{\chi} \in \operatorname{Esd}_{k} \sigma$ is vol $\sigma_{\chi}=\operatorname{vol} \sigma / k^{d}$.
Proof. By definition of the shape, vectors we have $P_{i}-$ $P_{0}=V_{i}+V_{i-1}+\ldots+V_{1}$. Using elementary column operations we find that volume can also be expressed using shape vectors:

$$
\operatorname{vol} \sigma=\frac{1}{d !} \cdot\left|\operatorname{det}\left(V_{1}, V_{2}, \ldots, V_{d}\right)\right| .
$$

Permuting the vectors does not affect the absolute value of the determinant, and shrinking them to $1 / k$-th their original lengths decreases the determinant by a factor of $1 / k^{d}$.

Simplex types. We have seen in the proof of the Independence Lemma that the shape vectors of a $d$ simplex in the edgewise subdivision are obtained by permuting and shrinking the shape vectors of $\sigma$. We use this to prove Property (2) of the Main Theorem.

Congruence Lemma. If the dimension of $\sigma$ is $d$ then the number of pairwise non-congruent $d$-simplices in $\operatorname{Esd}_{k} \sigma$ is at most $d ! / 2$.

Proof. Esd ${ }_{k} \sigma$ contains at most $d$ ! types of $d$-simplices, each defined by a permutation of the $d$ shape vectors of $\sigma$. Reversing the sequence (without reversing the directions of the vectors) effectively reflects the $d$-simplex through the origin. Reflected simplices are congruent, which implies the claimed upper bound.

In general the upper bound is tight. For sufficiently large $k$ all permutations occur, and for generic shape vectors two permutations define congruent $d$-simplices only if the permutations are equal or the reverse of each other. For special $d$-simplices $\sigma$ the congruence classes may further collapse. For example if every permutation of the $d$ shape vectors can be geometrically realized by an orthogonal transformation then we have only one congruence class. Such shape vectors are determined by their common length and the same angle between every pair of vectors. The angle can be chosen anywhere in the open interval between 0 and $\alpha_{d-1}$, where $\alpha_{d-1}$ is the angle formed by the vectors connecting the barycenter with two vertices of a regular $(d-1)$-simplex. For each angle in this interval we get a $d$-simplex that tiles $\mathbb{R}^{d}$ with congruent copies of itself. The 1-parameter family of such simplices was studied already by Moore [8]. According to Senechal [10] the problem of characterizing all $d$-simplices that tile $\mathbb{R}^{d}$ is open even for $d=3$.

Symmetry of dimension. Each face $\tau$ of $\sigma$ is a simplex which is subdivided as a consequence of the subdivision of $\sigma$. We prove Property (3) of the Main Theorem. 
FACE LEMma. Let $\tau$ be a face of $\sigma$ and assume the vertices of $\tau$ are ordered as specified by the ordering of the vertices of $\sigma$. Then $\operatorname{Esd}_{k} \tau \subseteq \operatorname{Esd}_{k} \sigma$.

Proof. Let $\Sigma=\{0,1, \ldots, d\}$ and $T \subset \Sigma$ such that $P_{i}$ is a vertex of $\tau$ iff $i \in T$. A point $X=\sum b_{i} \cdot P_{i} \in \sigma$ belongs to $\tau$ iff $b_{i}=0$ for all $i \in \Sigma-T$. As a consequence, the color scheme $\chi$ defined by $X$ contains only colors in $T$. Every color scheme with colors drawn from $T$ can be obtained from a color scheme with colors drawn from $\Sigma$ by dropping all columns that contain colors from $\Sigma-T$.

Symmetry of scale. It is plausible that a finer edgewise subdivision of $\sigma$ is a subdivision of a coarser edgewise subdivision provided this is true for the edges of $\sigma$. We prove Property (4) of the Main Theorem.

REFinement Lemma. Esd $\operatorname{Esd}_{k} \sigma=\operatorname{Esd}_{k \ell} \sigma$.

Proof. We show that every simplex in $\operatorname{Esd}_{k \ell} \sigma$ belongs to Esd $\ell$ of a simplex in $\operatorname{Esd}_{k} \sigma$. Since Esd $\operatorname{Es}_{k} \sigma$ and $\operatorname{Esd}_{\ell} \operatorname{Esd}_{k} \sigma$ both subdivide $\sigma$ this implies that the two subdivisions are the same.

Let $X=\sum b_{\imath} \cdot P_{i}$ be a point in $\sigma$, and let $B_{i}$ be the sum of the first $i$ barycentric coordinates, as usual. Let $\chi=\chi(X, k)$ and $\chi^{\prime}=\chi(X, k \ell)$ be the corresponding color schemes. The coordinates of the dividing lines in the corresponding stacks are $k \cdot B_{i} \bmod 1$ and $k \ell$. $B_{i} \bmod 1$. Equivalently, if $C$ is a dividing line for $\chi$ then $\ell \cdot C \bmod 1$ is a dividing line for $\chi^{\prime}$. In other words, the stack of $\chi^{\prime}$ is obtained from that of $\chi$ by chopping each rectangle into $\ell$ pieces, stacking the pieces, and expanding by $\ell$ in the horizontal direction for clarity. It follows that $\sigma_{\chi^{\prime}} \in \operatorname{Esd}_{\ell} \sigma_{\chi}$.

Symmetry of location. Ignoring boundary effects, the neighborhoods of any two vertices in an edgewise subdivision are the same. We prove Property (5) of the Main Theorem.

Translation Lemma. Let $P, P^{\prime}$ be vertices and $\sigma^{\prime}$ a simplex in $\operatorname{Esd}_{k} \sigma$. Then $\sigma^{\prime}+\left(P-P^{\prime}\right)$ either belongs to $\operatorname{Esd}_{k} \sigma$ or its interior lies outside $\sigma$.

Proof. Consider a point $X$ in the interior of $\sigma^{\prime}$ and assume $Y=X+\left(P-P^{\prime}\right)$ lies within $\sigma$. The coordinates of $P-P^{\prime}$ are integer multiples of $1 / k$. It follows that the vertical dividing lines of the stacks defined by $X$ and $Y$ are the same, but the rows defining the dividing lines may be different. Specifically, the row indices increase or decrease depending on the coordinates of $P-P^{\prime}$. The color scheme $\chi=\chi(Y, k)$ differs from $\chi^{\prime}=\chi(X, k)$ by increasing or decreasing colors accordingly. Since all points $X$ in the interior of $\sigma^{\prime}$ define the same color scheme, all $Y=X+\left(P-P^{\prime}\right)$ define the same transformed color scheme and therefore belong to the interior of the same simplex in the edgewise subdivision. This simplex is $\sigma^{\prime}+\left(P-P^{\prime}\right)$.

\section{Examples}

This section presents examples of the edgewise subdivision for simplices of dimension $2,3,4$.

Triangle. Figure 4 shows the edgewise subdivisions $\mathrm{Esd}_{2}$ and $\mathrm{Esd}_{3}$ of a triangle. The three vertices are

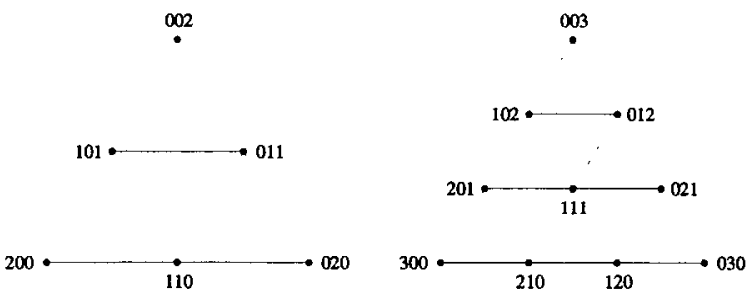

Figure 4: 4-division to the left and 9-division to the right.

labeled $k 00,0 k 0,00 k$ indicating the sequence used in the construction of the subdivisions. Not that the sequence matters, however, since all $6=3$ ! permutations lead to the same subdivision consisting of $k^{2}$ similar triangles. The vertex labels encode barycentric coordinates:

$$
x y z=\frac{x}{k} \cdot k 00+\frac{y}{k} \cdot 0 k 0+\frac{z}{k} \cdot 00 k .
$$

8-division of tetrahedron. The smallest non-trivial edgewise subdivision of a tetrahedron $\sigma$ is the 8-division illustrated in Figure 5. The tetrahedra adjacent to the

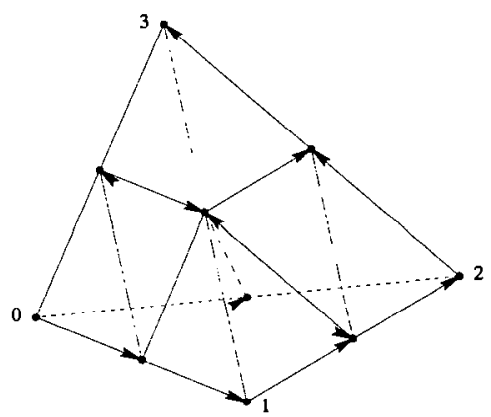

Figure 5: The octahedron in the center of the 8-division is decomposed into four tetrahedra surrounding the space diagonal.

four vertices are similar to $\sigma$. The four tetrahedra subdividing the center octahedron are generally not similar to $\sigma$ because their shape vectors come in a different 
sequence. Observe that exactly one diagonal of the octahedron belongs to the 8-division. Every proper subset $T \subset\{0,1,2,3\}$ defines a non-trivial partition into two sets and a halfway plane of points with barycentric coordinates $\sum_{i \in T} b_{i}=\sum_{j \notin T} b_{j}$. There are 7 halfway planes, and the 8-division can be defined by cutting $\sigma$ with 6 of them. The only halfway plane not used is defined by $T=\{0,2\}$ and crosses the diagonal that belongs to the subdivision.

27-division of tetrahedron. The 27-division of $\sigma$ shares many of the features of the 8-division; see Figure 6. It embeds four 8-divisions, one adjacent to every

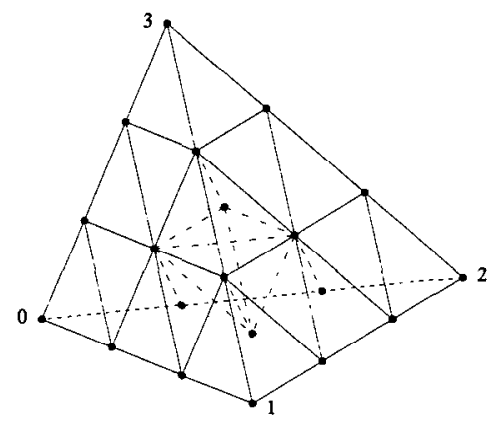

Figure 6: The 27-division contains four octahedra arranged around the tetrahedron in the center, each decomposed into four tetrahedra around parallel space diagonals.

vertex of $\sigma$. Any two such 8-divisions overlap in a single tetrahedron adjacent to the middle third of the edge joining the two vertices. We have $4: 8-\left(\begin{array}{l}4 \\ 2\end{array}\right)=26$, which shows that the four 8-divisions cover all tetrahedra except the one in the center of the 27-division.

16-division of 4-simplex. We are not able to draw the entire 16-division, but we can draw the 4-simplex $\sigma$ and argue about its 16-division. A 3-simplex is usually drawn as a convex quadrilateral in $\mathbb{R}^{2}$ simultaneously subdivided into two triangles in the two ways possible. Two of the triangles are in the back and the other two are in the front. By analogy we think of a 4 -simplex as a convex double tetrahedron in $\mathbb{R}^{3}$ simultaneously subdivided into two tetrahedra and into three tetrahedra. Maybe the three tetrahedra are in the front and the two are in the back. Figure 7 shows two copies of the 4simplex with front tetrahedra 1203, 2403, 4103 and back tetrahedra 0124,3124 . For $k=2$ the color scheme of an edge is a 2-by-2 matrix with entries from $\{0,1,2,3,4\}$. At least one of the colors is not used, which implies that all edges in the 16-division belong to the subdivisions of proper faces. We can therefore draw all edges by superimposing the edges of the five 8-divisions in the boundary of $\sigma$.
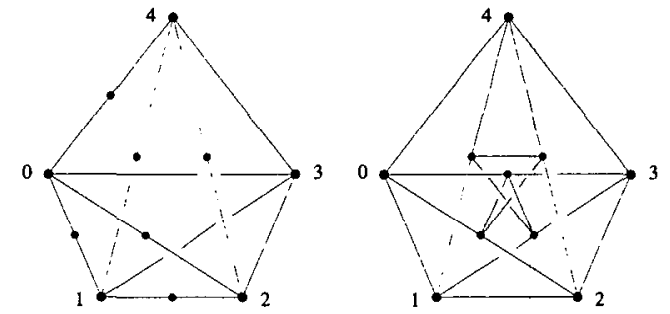

Figure 7: Two views of a 4-simplex. To the left the dotted lines show the 8-division of one tetrahedral face. To the right the dotted lines decompose a Möbius strip in the interior of the 4-simplex into five triangles.

The simplices that decompose the interior of $\sigma$ correspond to color schemes that use all five colors. There are only 5 triangles because there are only that many 2-by-3 matrices with five colors:

$$
\begin{gathered}
\left(\begin{array}{lll}
0 & 0 & 1 \\
2 & 3 & 4
\end{array}\right),\left(\begin{array}{lll}
0 & 1 & 1 \\
2 & 3 & 4
\end{array}\right),\left(\begin{array}{lll}
0 & 1 & 2 \\
2 & 3 & 4
\end{array}\right), \\
\left(\begin{array}{lll}
0 & 1 & 2 \\
3 & 3 & 4
\end{array}\right),\left(\begin{array}{lll}
0 & 1 & 2 \\
3 & 4 & 4
\end{array}\right) .
\end{gathered}
$$

As illustrated in Figure 7, the triangles form a Möbius strip one time around the center of $\sigma$. The five solid edges form the boundary of the strip and the five dotted edges cut it into five triangles. Similarly we find 20 tetrahedra that do not lie in the boundary and decompose $\sigma$ into $2^{4}=164$-simplices.

\section{Discussion}

This paper introduces the edgewise subdivision of a $d$ simplex and proves it is symmetric in dimension, in scale, and in location. The main ingredient in the construction is the abacus model of a simplex, which leads to a mechanical description of the subdivision amenable to computer implementations.

Variation. The abacus model can also be used to decompose a $d$-simplex into Cartesian products of simplices. Such decompositions are by-products of edgewise subdivisions. We return to the stack of rectangles as illustrated in Figure 2. Each rectangle contains dividers which are allowed to move freely between the left and the right end, except they cannot switch places. Each rectangle represents a simplex, and the stack represents the Cartesian product of these simplices. In $\mathbb{R}^{3}$ we have three types of 3-dimensional Cartesian products: tetrahedra, triangular prisms, and parallelepipeds. Figure 8 illustrates the decomposition for $k=3$, which is the smallest integer for which all three types arise. Compare this with the 27-division in Figure 6. 


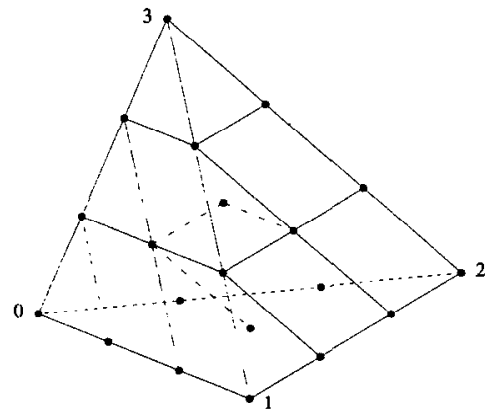

Figure 8: The tetrahedron is decomposed into three tetrahedra, six triangular prisms, and one parallelepiped. Each bounding triangle is decomposed into three triangles and three rhombi.

Questions. For $d=2$ there is only one edgewise subdivision per triangle and per integer $k \geq 1$. For $d=3$ there are three different edgewise subdivisions for each $k$, each one defined by the cyclic rotations and reversals of a permutation of the 4 vertices. How many edgewise subdivisions are there for general $d>3$ ? A related question is the identification of the best edgewise subdivision for a given measure of simplex shape. Particular measures have bcen studied by Parthasarathy [9] and others. Which permutation of the vertices of a $d$-simplex defines the edgewise subdivision that maximizes the minimum or the average shape measure of its simplices?

\section{References}

[1] J. BEY. Tetrahedral grid refinement. Computing $\mathbf{5 5}$ (1995), 355-378.

[2] H. S. M. COXETER. Discrete groups generated by reflections. Ann. of Math. 6 (1934), 13-29.

[3] T. DeRose, M. Kass and T. Truong. Subdivision surfaces in character animation. Computer Graphics, Proc. SIGGRAPH 1998, 85-94.

[4] H. Freudenthal. Simplizialzerlegung von beschränkter Flachheit. Ann. Math. 43 (1942), 580-582.

[5] D. R. GRAYSON. Exterior power operations in higher K-theory. K-Theory 3 (1989), 247-260.

[6] H. W. Kunn. Some combinatorial lemmas in topology. IBM J. Res. Dev. 45 (1960), 518-524.

[7] A. Liu AND B. JoE. Quality local refinement of tetrahedral meshes based on 8-subtetrahedron subdivision. Math. Comput. 65 (1996), 1183-1200.

[8] D. W. Moore. Simplicial Mesh Generation with Applications. Rept. 92-1322, Dept. Comput. Sci., Cornell Univ., Ithaca, New York, 1992.
[9] V. N. Parthasarathy. On tetrahedron shape distortion measures. Internat. J. Numer. Methods Engrg., 1991.

[10] M. Senechal. Which tetrahedra fill space? Math. Mag. 54 (1981), 227-243. 